\title{
Radiology Case Reports
}

\section{Malignant rhabdoid tumor of the liver: Findings at US, CT, and MRI, with histopathologic correlation}

\author{
Ho Nguyen, MD; Adam Stelling, MD; Alan Kuramoto, MD; Chirag Patel, MD; and Jennifer Keller, \\ MD
}

Malignant rhabdoid tumors are aggressive malignancies that can rarely present as hepatic masses in the pediatric population. The imaging findings are often nonspecific and usually suggest other more common hepatic tumors. We report the radiologic appearance of malignant rhabdoid tumor in a 3-monthold female with pathologic correlation.

\section{Introduction}

Malignant rhabdoid tumor, initially reported in the kidneys and thought to be a variant of Wilms tumor, is an uncommon and highly aggressive tumor with a poor prognosis. Ever since its recognition as a distinct clinical entity, it has been described in the brain, chest, and extrarenal abdominopelvic organs. We report a case of primary hepatic malignant rhabdoid tumor, present the imaging and pathology findings, and briefly discuss the differential diagnosis.

\section{Case report}

A 3-month-old female presented to her primary care physician with new-onset bilious emesis. Her family noticed a "funny shape" to her abdomen. Past medical history was positive for maternal gestational diabetes mellitus and transient neonatal jaundice. On physical exam, her abdomen was asymmetric, with firm swelling in the right upper quadrant.

Citation: Nugyen $\mathrm{H}$, Stelling A, Kuramoto A, Patel C, Keller J. Malignant rhabdoid tumor of the liver: Findings at US, CT, and MRI, with histopathologic correlation. Radiology Case Reports. (Online) 2014;1;854

Copyright: ( $\odot 2014$ The Authors. This is an open-access article distributed under the terms of the Creative Commons Attribution-NonCommercial-NoDerivs 2.5 License, which permits reproduction and distribution, provided the original work is properly cited. Commercial use and derivative works are not permitted.

Drs. Nguyen, Kuramoto, and Patel are in the Department of Radiology, Dr. Stelling is in the Department of Pathology and Laboratory Medicine, and Dr. Keller is in the Department of Surgery, all at University of California Davis Medical Center, Sacramento CA. Contact Dr. Nguyen at ho.nguyen@ucdmc.ucdavis.edu.

Competing Interests: The authors have declared that no competing interests exist. DOI: $10.2484 /$ rcr.v9i1.854
Abdominal ultrasound demonstrated a large, complex cystic mass with unclear organ of origin (Fig. 1). Subsequent

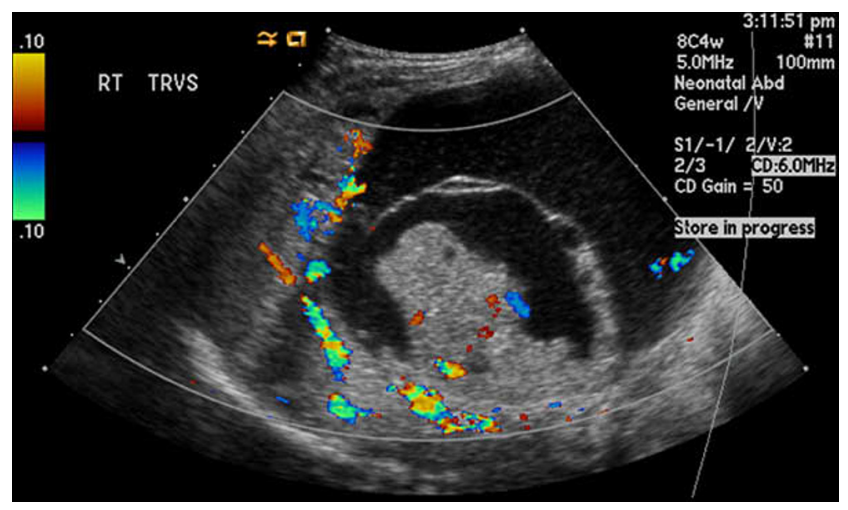

Figure 1. Color Doppler ultrasound image of the abdomen demonstrates a complex cystic mass of unclear organ of origin.

CT confirmed a large, septated, predominantly cystic mass within the right hepatic lobe (Fig. 2, A and B). The posterior margin of the mass was ill-defined. The mass displaced, but did not invade, the surrounding bowel and retroperitoneal structures. On MRI, there was a mildly enhancing solid component at the posterior-inferior aspect of the mass (Fig. 3). Alpha-fetoprotein was mildly elevated at $678 \mathrm{ng} / \mathrm{ml}$ (age-adjusted reference range of $3-417$ $\mathrm{ng} / \mathrm{ml}$ ). As the mass was mostly cystic, the primary consideration was mesenchymal hamartoma. The presence of a solid enhancing component suggested malignant degeneration or an undifferentiated embryonal sarcoma. 


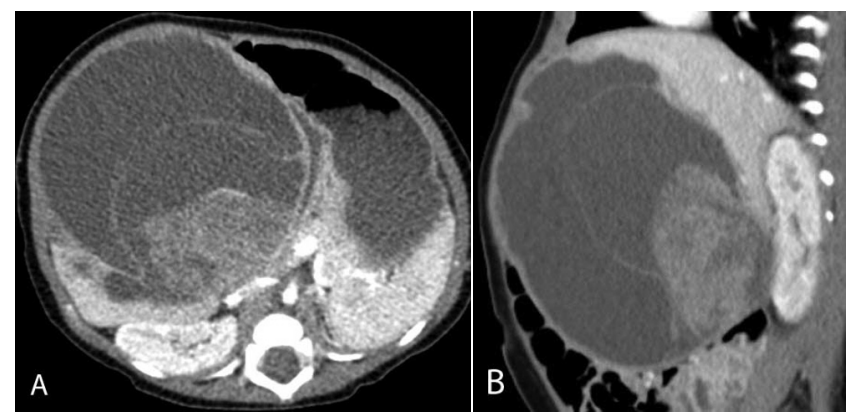

Figure 2, A and B. Axial and sagittal contrast-enhanced CT images delineate the intrahepatic location of the mass, which causes displacement of the surrounding peritoneal and retroperitoneal structures. The posterior margin is irregular and ill-defined.

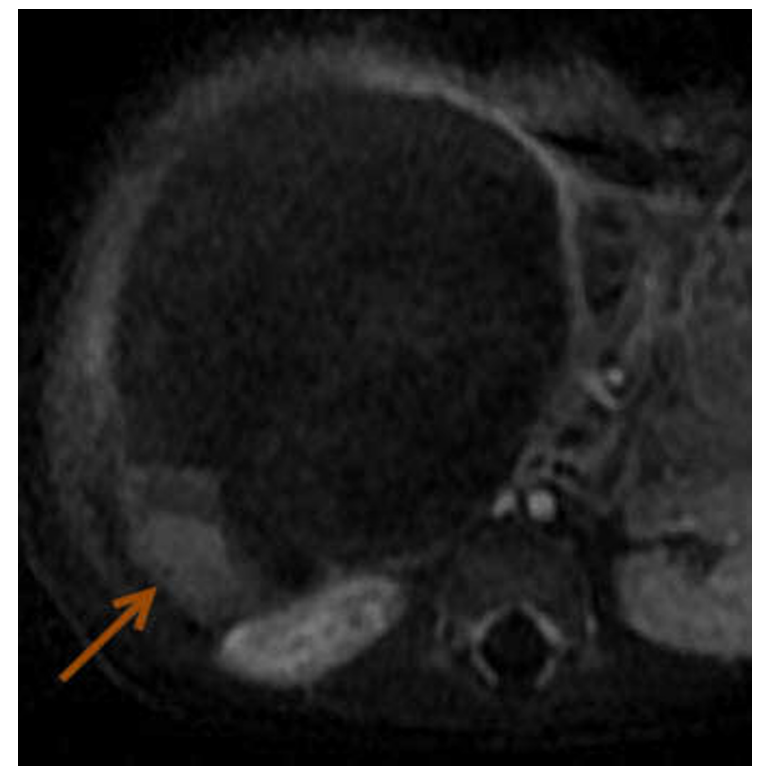

Figure 3. Axial MRI subtraction image demonstrates a mildly enhancing component (arrow) along the posterior margin of the hepatic mass.

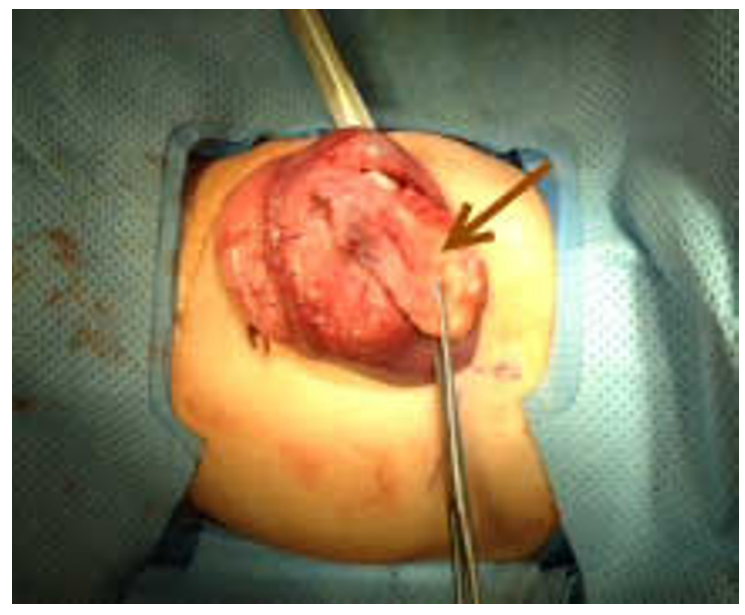

Figure 4. Intraoperative photo of the mass (arrow).
At laparotomy, a cystic mass was seen arising from the inferior right hepatic lobe without invasion of adjacent organs (Fig. 4). More than $200 \mathrm{ml}$ of thick yellow-brown fluid was drained. The superior and lateral walls of the mass were clearly separated from normal hepatic parenchyma. Medially, the mass extended almost to the level of the falciform ligament. A solid component was identified along the posterior-lateral wall of the mass. This portion of the mass could not be completely dissected from hepatic parenchyma.

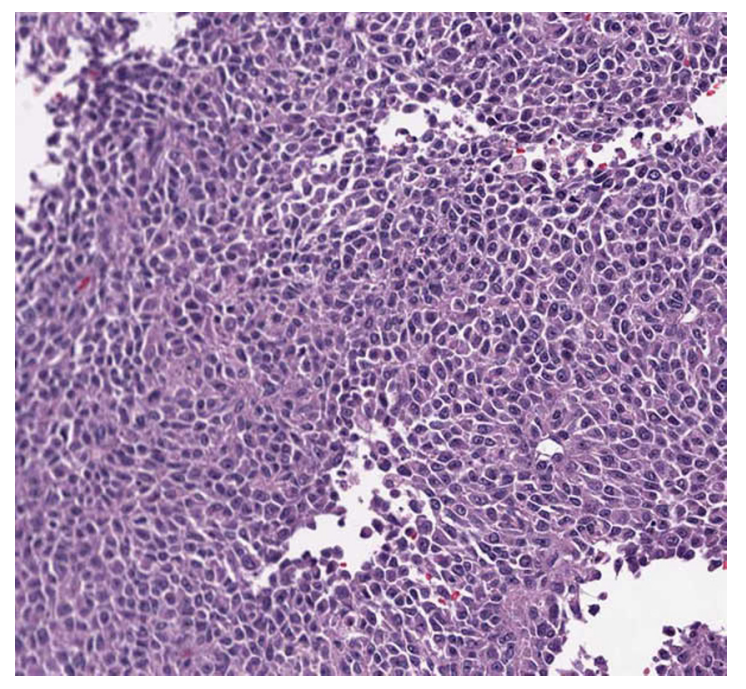

Figure 5. Histologic slide reveals an area filled entirely with epithelioid cells that demonstrate rhabdoid morphology (polygonal shape, dense eosinophilic cytoplasm, and eccentric nuclei).

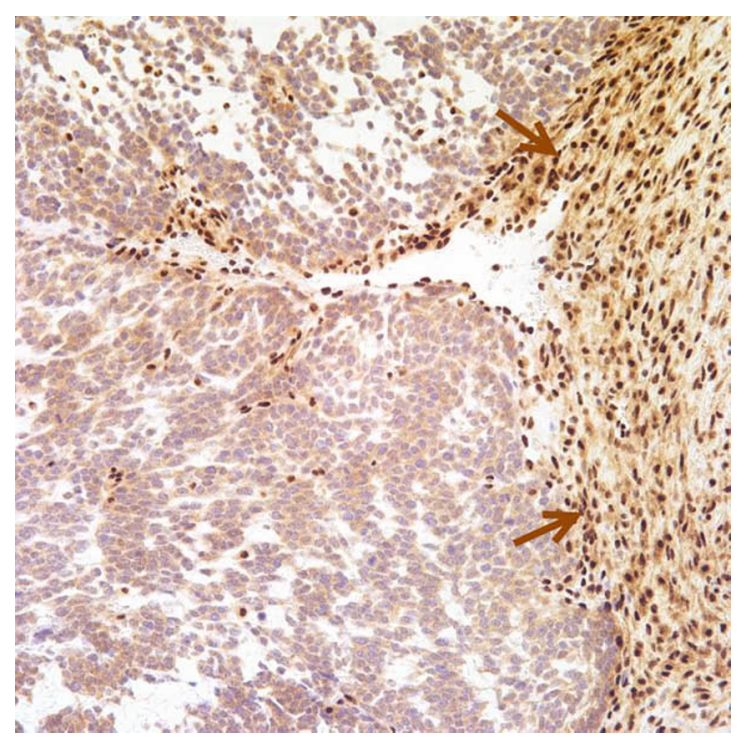

Figure 6. Immunohistochemical stain for INI-1 demonstrates diffuse loss of expression of this protein in the tumor cell nuclei, in contrast to strong INI-1 nuclear staining in the fibrous tissue to the right (arrows). 


\section{Malignant rhabdoid tumor of the liver: Findings at US, CT, and MRI, with histopathologic correlation}

Histologic sections revealed a heterogeneous and highly cellular mass with hyperchromatic nuclei and frequent mitoses. There were areas of fibrosis and cystic changes. The mass also contained epithelioid cells with rhabdoid morphology (Fig. 5). Immunohistochemistry showed lack of muscle markers and diffuse loss of nuclear INI-1 protein expression (Fig. 6). These features are most compatible with malignant rhabdoid tumor. Further workup demonstrated no evidence of metastatic disease. The patient was started on chemotherapy and underwent right hepatic lobectomy.

\section{Discussion}

Malignant rhabdoid tumors were originally described as a highly aggressive variant of Wilms tumors (1). They were so named due to their histologic resemblance to rhabdomyoblasts. Subsequent studies failed to confirm myogenic differentiation (2). Following lack of response to standard treatment regimens for Wilms tumors, they gained recognition as a distinct entity (3). Since then, malignant rhabdoid tumors have also been reported in extrarenal locations, including the central nervous system, liver, pelvis, chest, jejunum, and adrenal glands. The precise histiogenesis has not yet been elucidated (4).

Primary hepatic malignant rhabdoid tumors are extremely rare. To the best of our knowledge, 28 cases have been previously reported in the literature. The median age of presentation was 8 months, with most patients below 2 years of age. Clinically, patients are often asymptomatic until the lesions become large. Symptoms are nonspecific and include abdominal pain, anorexia, and weight loss (4, 5). On imaging, malignant rhabdoid tumors are large and heterogeneous. In one review of the imaging features of 14 cases, the median long-axis measurement was $10.6 \mathrm{~cm}(5)$. Occasionally, cystic changes and calcifications are present. In cases of rupture, peritumoral hematoma can be seen $(4,5)$. Due to their nonspecific imaging findings and rarity, diagnostic considerations are often given to more common hepatic masses. Alpha-fetoprotein is normal in most cases.

The differential diagnosis for a hepatic malignant rhabdoid tumor primarily includes hepatoblastoma, hepatocellular carcinoma, and undifferentiated embryonal sarcoma. Hepatoblastoma occurs in young patients, with $70 \%$ of cases occurring in children less than 2 years old. On imaging, hepatoblastomas usually appear as heterogeneously enhancing solid masses. Alpha-fetoprotein is elevated in more than 90 percent of cases. In our patient, the mass was predominantly cystic; the alpha-fetoprotein level was only mildly elevated, below the level typically seen with hepatoblastoma (6). Hepatocellular carcinoma is usually seen in older children (5-15 yo); elevated alpha-fetoprotein occurs in half of these patients. Undifferentiated embryonal sarcoma (also known as malignant mesenchymoma) is most often seen in older children and is not associated with elevated alpha-fetoprotein. When the mass is predominantly cystic, the possibility of mesenchymal hamartomas may be considered. These are seen in young children who have normal alpha-fetoprotein levels. Although these masses are benign, they can rarely undergo malignant degeneration (7).

On histology, this patient's hepatic mass was hypercellular and pleomorphic. It contained regions of small round blue cells and large epithelioid cells with rhabdoid morphology. The diagnosis was confirmed by immunohistochemistry. The cells were negative for alpha-fetoprotein, a marker that is seen with hepatoblastoma and hepatocellular carcinoma. In a pattern typical of rhabdoid tumors, the cells demonstrated a lack of muscle markers and a loss of nuclear INI-1 protein. The latter is reasonably specific for rhabdoid tumor, due to its association with mutations of the tumor-suppressor gene hSNF5/INI-1. Treatment options for rhabdoid tumors include surgery, chemotherapy, liver transplant, or a combination of these. Unfortunately, 70 percent of patients have metastatic disease on initial presentation, most commonly in the lungs and lymph nodes. The prognosis is generally poor, with a median survival time of 2 months (5).

We present the radiologic and pathologic features of hepatic malignant rhabdoid tumor in an infant. Although rare, it should be recognized as an aggressive malignancy with dismal prognosis and high risk of metastatic disease. This case underscores the importance of considering this entity when the combination of imaging, clinical, and laboratory findings does not fit one of the more common hepatic lesions.

\section{References}

1. Beckwith JB, Palmer NF. Histopathology and prognosis of Wilms tumors: results from the First National Wilms' Tumor Study. Cancer. 1978;41(5):1937-48. [PubMed]

2. Peng HQ, Stanek AE, Teichberg S, Shepard B, Kahn E. Malignant rhabdoid tumor of the kidney in an adult: a case report and review of the literature. Arch Path \& Lab Med. 2003;127(9):e371-3. [PubMed]

3. Palmer NF, Sutow W. Clinical aspects of the rhabdoid tumor of the kidney: a report of the National Wilms' Tumor Study Group. Med and Ped Oncol. 1983;11(4):242-5. [PubMed]

4. Sibileau E, Moroch J, Teyssedou C, Aube C. Malignant rhabdoid tumors of the liver: an exceptional tumor in adults - a case report and literature review. Eur 7 Gastroenterol \& Hepat. 201 1;23(1):104-8. [PubMed]

5. Abe T, Oguma E, Nozawa K, et al. Malignant rhabdoid tumor of the liver: a case report with US and CT manifestation. Fap 7 Radiol 2009;27(10):462-5. [PubMed]

6. Blohm ME, Vesterling-Horner D, Calaminus G, Gobel U. Alpha 1-fetoprotein (AFP) reference values in infants up to 2 years of age. Ped Hematol \& Oncol. 1998;15(2):135-42. [PubMed]

7. Ramanujam TM, Ramesh JC, Goh DW, et al. Malignant transformation of mesenchymal hamartoma of the liver: case report and review of the literature. 7 Ped Surg. 1999;34(11):1684-6. [PubMed] 